\title{
NEW CLASSES OF HYPERCYCLIC TOEPLITZ OPERATORS
}

\author{
EVGENY ABAKUMOV, ANTON BARANOV, STÉPHANE CHARPENTIER, \\ AND ANDREI LISHANSKII
}

\begin{abstract}
We study hypercyclicity of Toeplitz operators in the Hardy space $H^{2}(\mathbb{D})$ with symbols of the form $R(\bar{z})+\varphi(z)$, where $R$ is a rational function and $\varphi \in H^{\infty}(\mathbb{D})$. We relate this problem to cyclicity of certain families of functions for analytic Toeplitz operators and give new sufficient conditions for hypercyclicity based on deep results of B. Solomyak.
\end{abstract}

\section{INTRODUCTION AND MAIN RESULTS}

Toeplitz operators are among the most important objects in operator theory. They have numerous applications in complex analysis, theory of orthogonal polynomials, mathematical physics, etc. Recall that for a function $\psi \in L^{\infty}(\mathbb{T})$ the Toeplitz operator $T_{\psi}: H^{2} \rightarrow H^{2}$ with the symbol $\psi$ is defined as $T_{\psi} f=P_{+}(\psi f)$, where $P_{+}$stands for the orthogonal projection from $L^{2}(\mathbb{T})$ onto $H^{2}$. As usual, $\mathbb{D}$ and $\mathbb{T}$ denote the unit disc and the unit circle, respectively. We recall that the Hardy space $H^{2}$ of the unit disc $\mathbb{D}$ consists of functions analytic in $\mathbb{D}$ whose Taylor coefficients are square summable, and $H^{\infty}$ is the space of all bounded analytic functions in $\mathbb{D}$.

In the present paper we study the hypercyclicity property for a class of Toeplitz operators on $H^{2}$. A continuous linear operator $T$ on a separable Banach space $X$ is said to be hypercyclic if there exists $x \in X$ such that the set $\left\{T^{n} x: n \in \mathbb{N}_{0}\right\}$ is dense in $X$ (here $\mathbb{N}_{0}=\{0,1,2, \ldots\}$ ). Toeplitz operators with antianalytic symbols were among the basic examples of hypercyclic operators. In 1969, S. Rolewicz [6] showed that the operator $T_{\alpha \bar{z}}$ (a multiple of the backward shift) is hypercyclic on $H^{2}$ whenever $|\alpha|>1$. Later, G. Godefroy and J. Shapiro [3] showed that for a function $\varphi \in H^{\infty}$ the antianalytic Toeplitz operator $T_{\bar{\varphi}}$ is hypercyclic if and only if $\varphi(\mathbb{D}) \cap \mathbb{T} \neq \emptyset$. On the other hand, it is obvious that there are no hypercyclic Toeplitz operators with analytic symbols (i.e., among multiplication operators).

However, the problem of describing hypercyclic Toeplitz operators in the general case seems to be largely open. This problem was explicitly stated by S. Shkarin [7] who described hypercyclic Toeplitz operators with symbols of the form $\Phi(z)=a \bar{z}+b+$

2020 Mathematics Subject Classification. 47A16, 47B35, 30H10.

Key words and phrases. hypercyclic operator, Toeplitz operator, univalent function.

The results of Sections 2 and 3 were obtained with the support of Ministry of Science and Higher Education of the Russian Federation, agreement No 075-15-2019-1619. The results of Section 4 were obtained with the support of Russian Science Foundation grant 19-11-00058. 
$c z$ (i.e., with tridiagonal matrix). A. Baranov and A. Lishanskii [1] gave necessary and (separately) sufficient conditions for hypercyclicity of Toeplitz operators with polynomial antianalytic part of the symbol, that is, for symbols $\Phi$ of the form $\Phi(z)=$ $P\left(\frac{1}{z}\right)+\varphi(z)$, where $P$ is a polynomial and $\varphi \in H^{\infty}$. It turned out that the valence of the symbol played the central role in this study.

In the present paper we will consider more general symbols. Let $R$ be a rational function without poles in $\overline{\mathbb{D}}$, i.e.,

$$
R(z)=P(z)+\sum_{l=1}^{r} \sum_{j=1}^{k_{l}} \frac{\alpha_{l, j}}{\left(z-\eta_{l}\right)^{j}},
$$

where $P(z)=\sum_{k=0}^{N_{1}} c_{k} z^{k}$ is a polynomial of degree $N_{1}, \alpha_{l, j} \in \mathbb{C}$, and $\eta_{l} \in \widehat{\mathbb{D}}:=\mathbb{C} \backslash \overline{\mathbb{D}}$, $1 \leq l \leq r$, are the (distinct) poles of $R$ of multiplicities $k_{l}$. We put $N_{2}=\sum_{l=1}^{r} k_{l}$ and denote by $N=N_{1}+N_{2}$ the degree of the rational function $R$. We do not exclude the cases when $N_{1}=0$ (i.e., $P=$ const) or $N_{2}=0(R=P)$. Finally, let $\varphi \in H^{\infty}$, and put

$$
\Phi(z)=R\left(\frac{1}{z}\right)+\varphi(z) .
$$

Thus, $\Phi$ is analytic in $\mathbb{D}$ except the poles $1 / \eta_{1}, \ldots, 1 / \eta_{r}$. The aim of this note is to describe new classes of hypercyclic Toeplitz operators $T_{\Phi}$ with the symbols of the form (2)).

As in [1, our conditions will be formulated in terms of the valence of $\Phi$. Recall that an analytic function $h$ in a domain $D$ is said to be $n$-valent in $D$ if the equation $h(z)=w$ has at most $n$ solutions in $D$ counting multiplicities for any $w \in \mathbb{C}$. It is said to be exactly $n$-valent in $D$ if this equation has exactly $n$ solutions (counting multiplicities) for any $w \in h(\mathbb{D})$. Note that $\Phi$ (given by (2) ) has a pole of order $N_{1}$ at zero and poles of multiplicities $k_{l}$ at $1 / \eta_{l}$, whence the equation $\Phi(z)=w$ has exactly $N$ solutions when $|w|$ is sufficiently large.

Let us first illustrate the connection between the valence of $\Phi$ and the hypercyclicity of $T_{\Phi}$ through a necessary condition. For the case of symbols with polynomial antianalytic part the statement was proved in [1].

Proposition 1.1. Let $\Phi$ be given by (2). If $T_{\Phi}$ is hypercyclic, then the function $\Phi$ is $N$-valent in $\mathbb{D}$.

Let us also note that if $T_{\Phi}$ is hypercyclic, then we have

$$
\sigma\left(T_{\Phi}\right) \cap \mathbb{T} \neq \emptyset, \quad \sigma\left(T_{\Phi}\right) \cap \widehat{\mathbb{D}} \neq \emptyset .
$$

The first condition is a basic property that holds for any hypercyclic operator [4, Theorem 5.6], while the second one follows from the fact that, for any Toeplitz operator, its spectral radius coincides with its norm [5, Part B, Ch. 4]. 
It can be proven that if $\Phi$ is $N$-valent, then $\sigma\left(T_{\Phi}\right)=\mathbb{C} \backslash \Phi(\mathbb{D}, N)$, where $\Phi(\mathbb{D}, N)$ consists of those $w \in \mathbb{C}$ for which $\Phi(z)=w$ has exactly $N$ solutions in $\mathbb{D}$. We refer to [1, Proposition 2.2] for the details. We do not know whether the hypercyclicity of $T_{\Phi}$ implies that $\sigma\left(T_{\Phi}\right) \cap \mathbb{D} \neq \emptyset$.

The key step of the proof of our sufficient conditions for hypercyclicity is an application of the following principle, already used in [1]: Hypercyclicity of $T_{\Phi}$ can be reduced to standard cyclicity of some analytic Toeplitz (i.e., multiplication) operator. We recall that a finite (or countable) family $U$ of functions in $H^{2}$ is said to be cyclic for $T_{h}, h \in H^{\infty}(\mathbb{D})$, if the family $\left\{h^{k} u: u \in U, k \geq 0\right\}$ is complete in $H^{2}$. More specifically, for $\lambda \in \mathbb{C} \backslash \overline{\Phi(\mathbb{D})}$, let us set

$$
h_{\lambda}(z)=\frac{1}{\Phi(z)-\lambda} .
$$

Clearly, $h_{\lambda}$ is analytic and bounded in $\mathbb{D}$.

Proposition 1.2. Let $\Phi$ be given by (2). We assume that

$$
\mathbb{D} \cap(\mathbb{C} \backslash \Phi(\mathbb{D})) \neq \emptyset \text { and } \widehat{\mathbb{D}} \cap(\mathbb{C} \backslash \Phi(\mathbb{D})) \neq \emptyset
$$

and that, for any $\lambda \in \mathbb{C} \backslash \overline{\Phi(\mathbb{D})}$, the family $\left\{1, z, \ldots, z^{N-1}\right\}$ is cyclic for $T_{h_{\lambda}}$ with $h_{\lambda}$ given by (3). Then $T_{\Phi}$ is hypercyclic.

Based on this important observation, we will then formulate several sufficient conditions for the hypercyclicity of $T_{\Phi}$. From now on, we assume that $\Phi$ is given by (2) and satisfies $(4)$. We denote by $A(\mathbb{D})$ the disc algebra, i.e., the space of functions continuous in $\overline{\mathbb{D}}$ and analytic in $\mathbb{D}$.

The following result concerning the case of constant and maximal valence of the symbol is a direct extension of [1, Theorem 1.2].

Theorem 1.3. Assume that $\varphi \in A(\mathbb{D})$ and $\Phi$ satisfies the following Maximal Valence Condition:

(MVC) the function $\Phi$ is exactly $N$-valent on $\overline{\mathbb{D}} \backslash\left\{0,1 / \eta_{1}, \ldots, 1 / \eta_{r}\right\}$, i.e., for any $w \in \Phi(\overline{\mathbb{D}})$ the equation $\Phi(z)=w$ has exactly $N$ solutions in $\overline{\mathbb{D}}$ counting multiplicities.

Then $T_{\Phi}$ is hypercyclic.

Theorem 1.3, however, does not apply if $\Phi$ has varying valence in $\overline{\mathbb{D}}$. A novel feature of the present note is that we make use of deep results of B. Solomyak about cyclic families for analytic Toeplitz operators. Together with Proposition 1.2, this allows us to significantly enlarge the class of symbols inducing hypercyclic Toeplitz operators and, in particular, to include symbols with varying valence. These results are new even for symbols with polynomial antianalytic part studied in [1].

Cyclicity of families of functions for analytic Toeplitz operator is very far from being understood; deep results in this direction were obtained by B. Solomyak [8] 
and B. Solomyak and A. Volberg [9] in 1980-s. We use the geometric conditions on the valence of the function $\Phi$ given in [8] to distinguish two more classes of hypercyclic Toeplitz operators.

Theorem 1.4. Assume that $\varphi \in A(\mathbb{D})$ and, for some $\lambda \in \mathbb{C} \backslash \overline{\Phi(\mathbb{D})}$, the function $h_{\lambda}$ given by (3) satisfies the Increasing Argument Condition:

(IAC) The set $h_{\lambda}(\mathbb{T})$ is a finite union of $C^{2}$-smooth Jordan arcs and some continuous branch of the function $t \mapsto \arg h_{\lambda}\left(e^{i t}\right)$ is (strictly) increasing on $[0,2 \pi]$.

Then $T_{\Phi}$ is hypercyclic.

To state the last (and, in a sense, more general) result, we need to introduce some notations (see [8, Paragraph 1.3]). Let $h$ be a meromorphic function in $\overline{\mathbb{D}}$ (i.e., meromorphic in $\{|z|<\rho\}$ for some $\rho>1$ ) with poles $\eta_{1}, \ldots, \eta_{s}$ in $\mathbb{D}$. Then the set $h(\mathbb{T})$ splits the plane into finitely many connected components. Let $\Omega_{h}^{(k)}$ be the union of the connected components of $\mathbb{C} \backslash h(\mathbb{T})$ in which the number of pre-images in $\mathbb{D} \backslash\left\{\eta_{1}, \ldots, \eta_{s}\right\}$ of $h$ is equal to $k$ (counting multiplicity). The bounded connected components of the set $\mathbb{C} \backslash \overline{h(\mathbb{D})}=: \Omega_{h}^{(0)}$ will be called holes.

We say that $h$ is a function of general position if it is analytic in a neighborhood of $\mathbb{T}$, the curve $t \mapsto h\left(e^{i t}\right)$ has only finitely many points of self-intersection which are simple and transversal, and $\left.h^{\prime}\right|_{\mathbb{T}} \neq 0$.

Finally, let us say that two disjoint open sets $U$ and $V$ in $\mathbb{C} \backslash h(\mathbb{T})$ are adjacent if $\bar{U} \cap \bar{V}$ contains $h(I)$ for some open arc $I \subset \mathbb{T}$. Then we remark that if $h$ is meromorphic in $\overline{\mathbb{D}}$ and of general position, then a connected component of $\Omega_{h}^{\left(k_{1}\right)}$ and a connected component of $\Omega_{h}^{\left(k_{2}\right)}$ are adjacent if and only if $k_{1}=k_{2}-1$ or $k_{1}=k_{2}+1$. Moreover, if for some $\lambda \in \Omega_{h}^{0}, h_{\lambda}$ is given by (3), then $h_{\lambda}$ is of general position if and only if so $\Phi$ is.

Theorem 1.5. Assume that $\Phi$ is of general position and satisfies the following Decreasing Valence Condition:

(DVC) For some $\lambda_{0} \in \mathbb{D} \cap \Omega_{\Phi}^{(0)}$ and $\lambda_{1} \in \widehat{\mathbb{D}} \cap \Omega_{\Phi}^{(0)}$, for any $j \in\{0,1\}$, and for any component $G \subset \Omega_{\Phi}^{(k)}, k \geq 1$, there exist connected components $G_{i}$ of $\Omega_{\Phi}^{(i)}, 1 \leq i \leq k$, such that $G=G_{k}$, the components $G_{i}$ and $G_{i-1}$ are adjacent for $1<i \leq k$, and $G_{1}$ is adjacent to the hole which contains $\lambda_{j}$.

Then $T_{\Phi}$ is hypercyclic.

We shall observe that under the assumption of the previous theorem, condition (DVC) also reads as follows: For some $\lambda_{0} \in \mathbb{D} \cap \Omega_{\Phi}^{(0)}$ and some $\lambda_{1} \in \widehat{\mathbb{D}} \cap \Omega_{\Phi}^{(0)}$, and for any $w \in \mathbb{C} \backslash \overline{\Phi(\mathbb{D})}$, there exist continuous paths $\gamma_{0}, \gamma_{1}:[0,1] \rightarrow \mathbb{C}$ with $\gamma_{i}(0)=w$ and $\gamma_{i}(1)=\lambda_{i}$ such that $\gamma_{i} \cap \Omega_{\Phi}^{(k)}$ is connected for any $k \geq 0$ and $i=0,1$. 
Remark 1.6. Let $\lambda_{0} \in \mathbb{D} \cap \Omega_{\Phi}^{(0)}$ and $\lambda_{1} \in \widehat{\mathbb{D}} \cap \Omega_{\Phi}^{(0)}$. Then it is easily seen that $\Phi$ satisfies (DVC) for $\lambda_{0}$ and $\lambda_{1}$ if and only if both functions $h=\frac{1}{\Phi-\lambda_{0}}$ and $h=\frac{1}{\Phi-\lambda_{1}}$ are analytic in $\overline{\mathbb{D}}$, are of general position, and satisfy the following condition:

(DVC') For any component $G \subset \Omega_{h}^{(k)}, k \geq 1$, there exist connected components $G_{i}$ of $\Omega_{h}^{(i)}, 1 \leq i \leq k$, such that $G=G_{k}$, the components $G_{i}$ and $G_{i-1}$ are adjacent for $1<i \leq k$, and $G_{1}$ is adjacent to the unbounded connected component of $\Omega_{h}^{(0)}$.

Pictures of domains having this property or not can be found in [8, Fig. 1, Page 812 ] and in Figures 1 and 2 below.

In the next section we provide examples of symbols $\Phi$ of the form (2) satisfying (MVC), (IAC) or (DVC). The proofs of Propositions 1.11 .2 and Theorems 1.3 1.5 are postponed to Sections 3 and 4

\section{EXAMPLES}

Example 2.1. Let $\Psi$ be a univalent function in $A(\mathbb{D})$ which maps $\mathbb{D}$ onto a Jordan domain $\Omega$ with $0 \in \Omega$ and let $B$ be a finite Blaschke product of degree $N$. Let $\gamma \in \mathbb{D}$ be such that $\Psi(\gamma)=0$. Then

$$
\Phi(z)=\frac{1}{\Psi \circ B(z)}=\sum_{l=1}^{r} \sum_{j=1}^{k_{l}} \frac{a_{l, j}}{\left(z-\lambda_{l}\right)^{j}}+\varphi(z),
$$

where $\lambda_{1}, \ldots \lambda_{l}$ are such that $B\left(\lambda_{l}\right)=\gamma$ with multiplicity $k_{l}$ and $\varphi \in A(\mathbb{D})$. Thus, $\Phi$ is a symbol of the form (2) which satisfies (MVC).

Example 2.2. Another example of a symbol satisfying (MVC) can be obtained as follows. Let $\Omega$ be a Jordan domain invariant under rotation by $2 \pi / N$, for some $N \in \mathbb{N}$. and let $\Psi: \mathbb{D} \rightarrow \Omega$ be the conformal mapping with $\Psi(0)=0$. Then it is clear that $\Phi=1 / \Psi^{N}$ will be exactly $N$-valent in $\mathbb{D}$.

Example 2.3. It is easy to provide with examples of $\Phi$ satisfying (IAC) as small perturbation of $z^{n}$. Indeed, let $\varepsilon>0, \lambda \in \mathbb{C}$ and $\Psi \in A(\mathbb{D}) \cap \mathcal{C}^{1}(\overline{\mathbb{D}})$. Then one defines

$$
\Phi(z)=\lambda+\left[z^{n}(1+\varepsilon \Psi(z))\right]^{-1} .
$$

Clearly, $\Phi$ is of the form $P(1 / z)+\varphi(z)$, and a direct computation gives that the derivative of $t \mapsto \arg h\left(e^{i t}\right)$ is equal to $n+\mathcal{O}(\varepsilon)$, where $h=(\Phi-\lambda)^{-1}$. So $\Phi$ satisfies (IAC) for any $\varepsilon$ small enough.

Example 2.4. Let us first build a 2-valent function $h \in H(\overline{\mathbb{D}})$, of general position, which satisfies (DVC') and has a zero of order 2 at 0 . To do so, let us first (the steps 
are illustrated in Figure 1) consider the conformal map $\Psi$ which sends $\mathbb{D}$ onto the sector of annulus $\Gamma$ given by

$$
\Gamma=\left\{z \in \mathbb{C}: r<|z|<R,-\frac{\pi}{4}+\alpha<\arg z<\pi-\alpha\right\}
$$

with $0<r<R<(2 \sqrt{2}-1) r, 0<\alpha<\frac{\pi}{8}$. Then step 2 consists in composing $\Psi$ with the map $(1+\varepsilon z) z^{2}-\beta(1+i)$, where $\varepsilon>0$ is very small and $\beta$ is such that $0<\tilde{c}<c<\tilde{a}<a<\tilde{b}<b<\tilde{d}<d$ (see Figure 1 for the definitions of $a, \tilde{a}, b, \tilde{b}, c, \tilde{c}, d, \tilde{d})$. Note that such $\beta$ exists because of the choice of $r$ and $R$. Thus, the resulting map is $g(z)=(1+\varepsilon \Psi(z)) \Psi(z)^{2}-\beta(1+i)$. There is, however, a small problem that $g$ is not analytic in $\overline{\mathbb{D}}$. To overcome it consider $g(\rho z)$ for some $\rho \in(0,1)$ close enough to 1 . Finally, put

$$
h(z)=g^{2}(\rho z)=\left[(1+\varepsilon \Psi(\rho z)) \Psi(\rho z)^{2}-\beta(1+i)\right]^{2} .
$$

The resulting domain $h(\mathbb{D})$ is shown in the last part of Figure 1 .

It is easily seen that $h$ is 2 -valent, of general position, has zero of order 2 at 0 and satisfies (DVC'). Note also that $(h-\mu)^{-1}$ also satisfies (DVC') for any $\mu \notin h(\overline{\mathbb{D}})$.

If we put $\Phi=1 / h$, then it is clear that $\Phi(z)=a_{1} z^{-2}+a_{2} z^{-1}+\varphi(z)$, where $\varphi$ is analytic in $\mathbb{D}$. Thus, we can take $\lambda_{0} \in \mathbb{D}$ so that $h_{\lambda_{0}}$ has (DVC') (for e.g., $\lambda_{0}=0$ ). It remains to find $\lambda_{1}$. Note, that

$$
h_{\lambda_{1}}=\frac{1}{\Phi-\lambda_{1}}=-\frac{1}{\lambda_{1}}-\frac{1}{\lambda_{1}^{2}\left(h-\lambda_{1}^{-1}\right)} .
$$

Thus, if we can choose $\lambda_{1} \in \widehat{\mathbb{D}}$ so that $1 / \lambda_{1} \notin h(\overline{\mathbb{D}})$ (which is possible for most of the choices of $r$ and $R$ ) we conclude that $\Phi$ is of general position and has (DVC). By construction, $\Phi$ satisfies neither (MVC) nor (IAC).

Example 2.5. Choosing the parameter $\beta$ in a good way we can modify the construction in Example 2.4 so that $0<\tilde{a}<a<\tilde{c}<c<\tilde{b}<b<\tilde{d}<d$. Then, repeating the construction, we build $\Phi$ of general position, which does not satisfy the condition (DVC') (see Figure 2).

\section{NeCESSARY CONDITIONS}

In this section we prove Proposition 1.1. The proof goes along the same lines as the proofs in [1] and we include it for the sake of completeness only. Assume that $\Phi$ is not $N$-valent and so, for some $\mu$, the equation $\Phi(z)=\mu$ has $N+1$ solutions $z_{1}, z_{2}, \ldots z_{N+1}$ in $\mathbb{D}$ counting multiplicities. We assume that these solutions are distinct. If they are not distinct the argument requires an obvious modification (see [1 for details). We will show that the adjoint operator $T_{\bar{\Phi}}$ has an eigenvector and so $T_{\Phi}$ is not hypercyclic, a contradiction. 

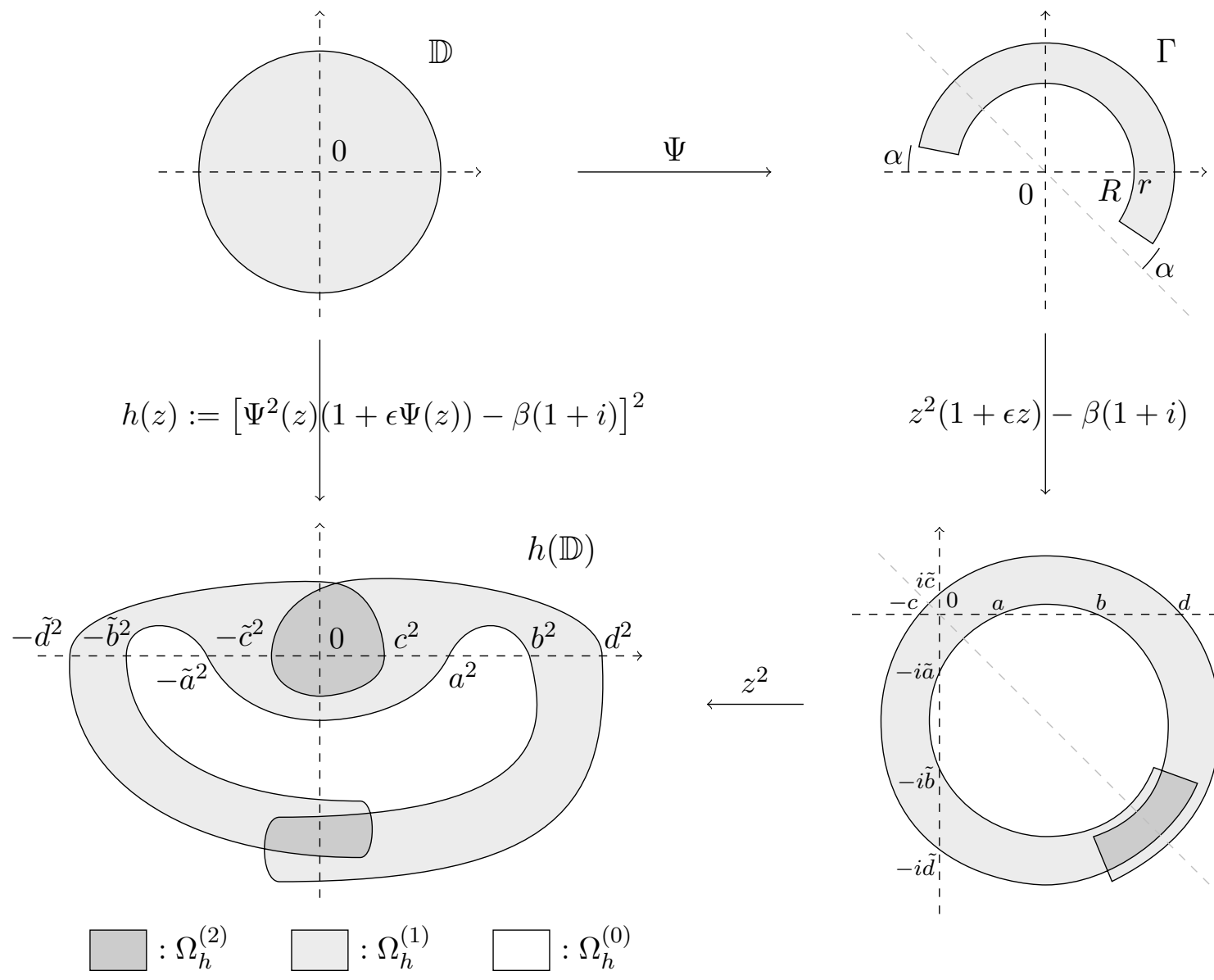

FIGURE 1. Example of $h$ satisfying (DVC')

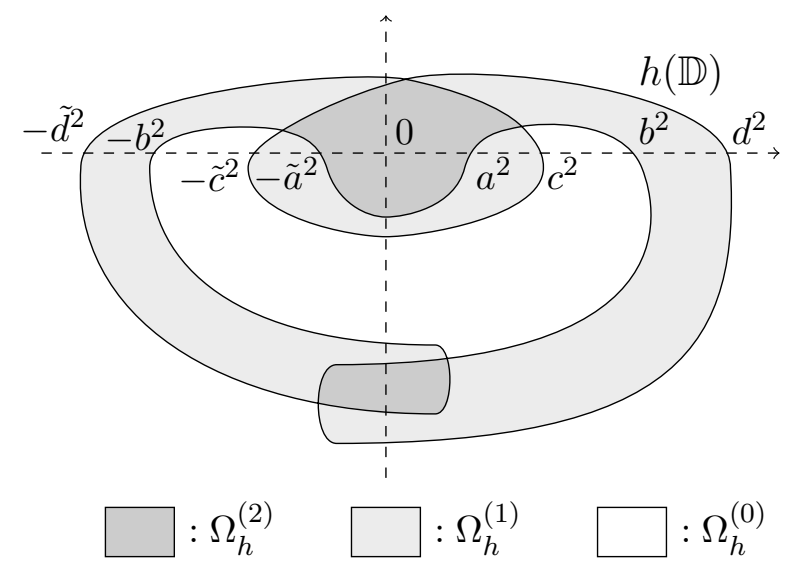

Figure 2. Example of $h$ which does not satisfy (DVC') 
We construct this eigenvector of $T_{\bar{\Phi}}$ as a linear combination of Cauchy kernels $k_{z_{m}}$, where $k_{\lambda}(z)=\frac{1}{1-\bar{\lambda} z}$. Recall that for any antianalytic Toeplitz operator we have $T_{\bar{\varphi}} k_{\lambda}=\overline{\varphi(\lambda)} k_{\lambda}$.

Put $f=\sum_{m=1}^{N+1} \beta_{m} k_{z_{m}}$, where $\beta_{m}$ are some complex coefficients. Note that $T_{\bar{\Phi}}=$ $T_{R^{*}(z)+\overline{\varphi(z)}}$, where

$$
R^{*}(z)=\sum_{k=0}^{N_{1}} \bar{c}_{k} z^{k}+\sum_{l=1}^{r} \sum_{j=1}^{k_{l}} \frac{\bar{\alpha}_{l, j}}{\left(z-\bar{\eta}_{l}\right)^{j}}
$$

is analytic and bounded in $\mathbb{D}$. Hence, using the fact that $R\left(1 / z_{m}\right)+\varphi\left(z_{m}\right)=\mu$, we get

$$
\begin{aligned}
T_{\bar{\Phi}} f(z)=\sum_{m=1}^{N+1} \beta_{m}\left(\frac{R^{*}(z)}{1-\overline{z_{m}} z}\right. & \left.+\frac{\overline{\varphi\left(z_{m}\right)}}{1-\overline{z_{m}} z}\right) \\
& =\bar{\mu} f(z)+\sum_{m=1}^{N+1} \beta_{m} \frac{R^{*}(z)-\overline{R\left(1 / z_{m}\right)}}{1-\overline{z_{m}} z} .
\end{aligned}
$$

Clearly,

$$
\frac{R^{*}(z)-\overline{R\left(1 / z_{m}\right)}}{1-\overline{z_{m}} z} \in \operatorname{span}\left\{z^{k}, \frac{1}{\left(z-\bar{\eta}_{l}\right)^{j}}: 0 \leq k \leq N_{1}-1,1 \leq l \leq r, 1 \leq j \leq k_{l}\right\} .
$$

Since the dimension of this span is $N$, we can find nontrivial $\beta_{m}$ such that the last sum in (5) is identically zero, and so $T_{\bar{\Phi}} f=\bar{\mu} f$.

\section{Sufficient CONDitions}

The proofs of Theorems 1.3 1.5 are each based on Proposition 1.2, which in turns is based on an application of the Godefroy-Shapiro Criterion (see [3], [2, Corollary 1.10] or [4, Theorem 3.1]).

Theorem (Godefroy-Shapiro Criterion). If, for a bounded linear operator $T$ on a separable Banach space $X$, both $\cup_{|\lambda|<1} \operatorname{Ker}(T-\lambda I)$ and $\cup_{|\lambda|>1} \operatorname{Ker}(T-\lambda I)$ span a dense subspace in $X$, then $T$ is hypercyclic.

We will then first search for the eigenvectors of $T_{\Phi}$.

4.1. Computation of eigenfunctions. Let us denote by $S_{n}(g)$ the $n$th partial sum of the Taylor series of $g \in H^{2}$ at 0 . Put $Q(z)=\prod_{l=1}^{r}\left(1-\eta_{l} z\right)^{k_{l}}$.

We recall that $T_{\bar{z}}$ is the backward shift $S^{*}$ on $H^{2}$, i.e.,

$$
T_{\bar{z}} f=S^{*} f=\frac{f(z)-f(0)}{z} \text { and } T_{\bar{z}^{k}} f=\left(S^{*}\right)^{k} f=\frac{f(z)-S_{k-1}(f)}{z^{k}} .
$$


Then, for $\eta \in \widehat{\mathbb{D}}, k \geq 1$ and $g \in H^{2}$,

$$
\begin{aligned}
\left(S^{*}-\eta I\right)^{k} g & =\sum_{j=0}^{k}\left(\begin{array}{l}
k \\
j
\end{array}\right)(-\eta)^{k-j}\left(S^{*}\right)^{j} g \\
& =g \sum_{j=0}^{k} \frac{\left(\begin{array}{l}
k \\
j
\end{array}\right)(-\eta)^{k-j}}{z^{j}}-\sum_{j=1}^{k}\left(\begin{array}{l}
k \\
j
\end{array}\right)(-\eta)^{k-j} \frac{S_{j-1}(g)}{z^{j}} \\
& =\frac{(1-\eta z)^{k}}{z^{k}} g-\frac{1}{z^{k}} \sum_{j=1}^{k}\left(\begin{array}{c}
k \\
j
\end{array}\right)(-\eta z)^{k-j} S_{j-1}(g) .
\end{aligned}
$$

Thus, for $f \in H^{2}$, applying the previous to $g=\left[\left(S^{*}-\eta I\right)^{k}\right]^{-1} f$, we obtain

$$
\left[\left(S^{*}-\eta I\right)^{k}\right]^{-1} f=\frac{z^{k} f+p_{k}}{(1-\eta z)^{k}},
$$

where $p_{k}$ is some polynomial of degree at most $k-1$. It follows that

$$
\begin{aligned}
T_{\Phi} f & =\sum_{k=0}^{N_{1}} c_{k}\left(S^{*}\right)^{k} f+\sum_{l=1}^{r} \sum_{j=1}^{k_{l}} \alpha_{l, j}\left[\left(S^{*}-\eta_{l}\right)^{j}\right]^{-1} f+\varphi f \\
& =\sum_{k=0}^{N_{1}} \frac{c_{k} f+q_{k}}{z^{k}}+\sum_{l=1}^{r} \sum_{j=1}^{k_{l}} \alpha_{l, j} \frac{z^{j} f+p_{l, j}}{\left(1-\eta_{l} z\right)^{j}}+\varphi f \\
& =\Phi f+\sum_{k=1}^{N_{1}} \frac{q_{k}}{z^{k}}+\sum_{l=1}^{r} \sum_{j=1}^{k_{l}} \frac{\alpha_{l, j} p_{l, j}}{\left(1-\eta_{l} z\right)^{k_{l}}},
\end{aligned}
$$

where $p_{l, j}$ is a polynomial of degree at most $k_{l}-1$ and $q_{k}$ is a polynomial of degree at most $k-1$ (while $q_{0}=0$ ).

Therefore, the equation $T_{\Phi} f_{\lambda}=\lambda f_{\lambda}$ is equivalent, for some polynomials $p$ and $q$ with degree at most $N_{2}-1$ and $N_{1}-1$ respectively, to

$$
f_{\lambda}=\frac{z^{N_{1}} p+Q q}{z^{N_{1}} Q(\Phi-\lambda)} .
$$

Clearly, $f_{\lambda} \in H^{2}$ for any $\lambda \in \mathbb{C} \backslash \overline{\Phi(\mathbb{D})}$, and so $f_{\lambda}$ is an eigenvector of $T_{\Phi}$. Note that this holds true for any choice of the polynomials $p$ and $q$ with degree at most $N_{2}-1$ and $N_{1}-1$ respectively.

4.2. Proof of Proposition 1.2. By (44), we can find two open sets $U_{0} \subset \mathbb{D} \cap(\mathbb{C} \backslash$ $\overline{\Phi(\mathbb{D})})$ and $U_{1} \subset \widehat{\mathbb{D}} \cap(\mathbb{C} \backslash \overline{\Phi(\mathbb{D})})$ consisting of eigenvalues of $T_{\Phi}$. Let $\lambda_{0} \in U_{0}$. 
Expanding $f_{\lambda}$ around $\lambda_{0}$, for $\lambda$ close to $\lambda_{0}$, we get

$$
f_{\lambda}=\frac{z^{N_{1}} p+Q q}{Q z^{N_{1}}\left(\Phi-\lambda_{0}\right)} \sum_{k \geq 0} h^{k}\left(\lambda-\lambda_{0}\right)^{k},
$$

with $h=\frac{1}{\Phi-\lambda_{0}}$. Thus, if $f \in H^{2}$ is orthogonal to all such $f_{\lambda}$, then

$$
f \perp \frac{z^{N_{1}} p+Q q}{Q z^{N_{1}}\left(\Phi-\lambda_{0}\right)} h^{k}, \quad k \geq 0
$$

for any polynomials $p$ and $q$ with degree at most $N_{2}-1$ and $N_{1}-1$ respectively. Note that any polynomial of degree at most $N-1$ can be represented as $z^{N_{1}} p+Q q$ for some $p$ and $q$, whence

$$
f \perp\left(z^{N_{1}} Q\left(\Phi-\lambda_{0}\right)\right)^{-1} z^{j} h^{k}, \quad 0 \leq j \leq N-1, \quad k \geq 0 .
$$

Now, by assumption, the family $\left\{1, z, \ldots, z^{N-1}\right\}$ is a cyclic set for the operator $T_{h}$, i.e., the family $\left\{z^{j} h^{k}: 0 \leq j \leq N-1, k \geq 0\right\}$ is complete in $H^{2}$. Since $z^{N_{1}} Q\left(\Phi-\lambda_{0}\right)$ is a nonvanishing function in $A(\mathbb{D})$, it is invertible in $A(\mathbb{D})$, and so the family

$$
\left\{\left(z^{N_{1}} Q\left(\Phi-\lambda_{0}\right)\right)^{-1} z^{j} h^{k}: 0 \leq j \leq N-1, k \geq 0\right\}
$$

is also complete. Thus, $f=0$.

Since the same argument works for $U_{1}$, we see that the families $\left\{f_{\lambda}\right\}_{\lambda \in U_{0}}$ and $\left\{f_{\lambda}\right\}_{\lambda \in U_{1}}$ are complete in $H^{2}$. Hence, by the Godefroy-Shapiro Criterion, $T_{\Phi}$ is hypercyclic.

Remark 4.1. Note that for the conclusion of Proposition 1.2 to hold it is sufficient to assume that there exist $\lambda_{0} \in \mathbb{D} \cap(\mathbb{C} \backslash \overline{\Phi(\mathbb{D})})$ and $\lambda_{1} \in \widehat{\mathbb{D}} \cap(\mathbb{C} \backslash \overline{\Phi(\mathbb{D})})$ such that for $h_{0}=\frac{1}{\Phi-\lambda_{0}}$ and $h_{1}=\frac{1}{\Phi-\lambda_{1}}$ the family $\left\{1, z, \ldots, z^{N-1}\right\}$ is cyclic for $T_{h_{0}}$ and for $T_{h_{1}}$.

Before giving the proofs of Theorems 1.3 1.5, let us recall Solomyak's results that we shall apply.

4.3. Solomyak's theorems. Even if we use only a very special case of the results from [8], we find it appropriate to give a short survey of them. Recall that a matrixvalued function of size $n \times p$ whose entries are in $H^{2}$ is said to be outer if $p \geq n$ and the greatest common inner divisor of its minors of order $n$ is 1 . In this definition it is possible that $p=\infty$.

Theorem 4.2 (B. Solomyak, 1987). Let $h \in A(\mathbb{D})$ and let the set $h(\mathbb{T})$ be a finite union of $\mathcal{C}^{2}$-smooth Jordan arcs. If $h$ satisfies the property (IAC), then the set $\left\{u_{1}, \ldots, u_{m}\right\} \subset H^{2}$ is cyclic for the operator $T_{h}$ if and only if the following two conditions hold: 
(P1) For any $\zeta_{1}, \zeta_{2}, \ldots, \zeta_{l} \in \mathbb{D}$ such that $h\left(\zeta_{1}\right)=\ldots=h\left(\zeta_{l}\right)=a$, and the value a is taken at the point $\zeta_{j}$ with multiplicity $K_{j}$, we have

$$
\operatorname{rank}\left[u_{i}\left(\zeta_{j}\right), u_{i}^{\prime}\left(\zeta_{j}\right), \ldots u_{i}^{\left(K_{j}-1\right)}\left(\zeta_{j}\right) \substack{1 \leq i \leq m \\ 1 \leq j \leq l}_{\substack{1 \leq j \\ j=1}}^{l} K_{j}\right.
$$

(P2) For any $z \in \mathbb{T}$ and for sufficiently small neighbourhood $V_{z}$ such that in $V_{z} \cap \mathbb{D}$ there exist $k$ one-to-one branches $\psi_{1}=i d, \psi_{2}, \ldots, \psi_{k}$ of the function $h^{-1} \circ h$, the matrix-function $\left[\left(u_{i} \circ \psi_{j}\right)(\zeta)\right]_{1 \leq i \leq m, 1 \leq j \leq k}$ is outer in $V_{z} \cap \mathbb{D}$.

Note that this theorem applies also to the case when $\left\{u_{i}\right\}_{i}$ is an infinite sequence, i.e., $m=\infty$.

Theorem 4.3 (B. Solomyak, 1987). Let $h$ be analytic in $\overline{\mathbb{D}}$. If $h$ is of general position and satisfies the property (DVC'), then the set $\left\{u_{1}, \ldots, u_{m}\right\}, m \in \mathbb{N}$, is cyclic for the operator $T_{h}$ if and only if the conditions (P1), (P2) and (P3) hold, where

(P3) For any hole $G \subset \Omega_{h}^{(0)}$ there exist $i, j \leq m$ such that $\left(u_{i} \circ \nu^{-1}\right) /\left.\left(u_{j} \circ \nu^{-1}\right)\right|_{\partial G} \notin$ $N(G)$, where $\nu=\left.h\right|_{\mathbb{T}}$ and $N(G)$ is the Nevanlinna class in $G$.

We shall apply Solomyak's theorems only to the set $\left\{1, z, \ldots, z^{N-1}\right\}$ which, by [8, Sect. 1.3, Rem. 3], satisfies the conditions (P1), (P2) and (P3) for any $N$-valent $h$ satisfying the conditions of Solomyak's theorems 4.2 and 4.3 .

\subsection{Proof of Theorems $1.3-1.5$.}

Proof of Theorem 1.3. If $\Phi$ satisfies (MVC), then also for any $\lambda \in \mathbb{C} \backslash \overline{\Phi(\mathbb{D})}$ the function $h=\frac{1}{\Phi-\lambda}$ has the property that for any $w \in h(\overline{\mathbb{D}})$ the equation $h(z)=w$ has exactly $N$ solutions in $\overline{\mathbb{D}}$. By [1, Proposition 3.1] the family $\left\{z^{j} h^{k}: 0 \leq j \leq\right.$ $N-1, k \geq 0\}$ is complete in $H^{2}$. Thus, by Proposition $1.2, T_{\Phi}$ is hypercyclic.

Proof of Theorem 1.4. Since $\Phi$ satisfies (IAC), there is $\lambda \in \mathbb{C} \backslash \overline{\Phi(\mathbb{D})}$ such that Theorem 4.2 applies to the function $h=\frac{1}{\Phi-\lambda}$. Thus, the family $\left\{z^{j} h^{k}: 0 \leq j \leq N-1, k \geq\right.$ $0\}$ is complete in $H^{2}$. Let us show that for any other $\mu \in \mathbb{C} \backslash \overline{\Phi(\mathbb{D})}$ and $h_{\mu}=\frac{1}{\Phi-\mu}$ the family $\left\{z^{j} h_{\mu}^{k}: 0 \leq j \leq N-1, k \geq 0\right\}$ is also complete in $H^{2}$.

Note that $h_{1}=c_{1}+\frac{c_{2}}{h-c_{3}}$, where $c_{1}, c_{2}, c_{3}$ are some constants (which can be written explicitly in terms of $\lambda$ and $\mu$, see (3) ) and $c_{3} \notin h(\overline{\mathbb{D}})$. By condition (IAC), the closed domain $\Omega=h(\overline{\mathbb{D}})$ has no holes, and so the function $z$ can be approximated by functions in $\operatorname{span}\left\{\left(z-c_{3}\right)^{-k}: k \geq 0\right\}$ uniformly in $\Omega$. Thus, $h \in \overline{\operatorname{span}}\left\{\left(h-c_{3}\right)^{-k}\right.$ : $k \geq 0\}$ in $H^{2}$, whence

$$
\begin{aligned}
\overline{\operatorname{span}}\left\{z^{j} h_{1}^{k}: 0 \leq j \leq N\right. & -1, k \geq 0\} \\
& =\overline{\operatorname{span}}\left\{z^{j} h^{k}: 0 \leq j \leq N-1, k \geq 0\right\}=H^{2} .
\end{aligned}
$$


Now, $T_{\Phi}$ is hypercyclic by Proposition 1.2 .

Proof of Theorem 1.5 . Let $\lambda_{0} \in \mathbb{D} \cap(\mathbb{C} \backslash \overline{\Phi(\mathbb{D})})$ and $\lambda_{1} \in \widehat{\mathbb{D}} \cap(\mathbb{C} \backslash \overline{\Phi(\mathbb{D})})$. By Remark 1.6, both functons $h_{0}=\frac{1}{\Phi-\lambda_{0}}$ and $h_{1}=\frac{1}{\Phi-\lambda_{1}}$ belong to $A(\mathbb{D})$, are of general position and satisfy (DVC'). Then, by Theorem 4.3, the families $\left\{z^{j} h_{0}^{k}: 0 \leq j \leq N-1, k \geq 0\right\}$ and $\left\{z^{j} h_{1}^{k}: 0 \leq j \leq N-1, k \geq 0\right\}$ are complete in $H^{2}$. Therefore, by Proposition 1.2 and Remark 4.1, $T_{\Phi}$ is hypercyclic.

\section{REFERENCES}

[1] A. Baranov, A. Lishanskii, Hypercyclic Toeplitz operators, Results Math. 70 (2016), 3, 337347.

[2] F. Bayart, É. Matheron, Dynamics of Linear Operators, Cambridge University Press, 2009.

[3] G. Godefroy, J.H. Shapiro, Operators with dense, invariant cyclic vector manifolds, J. Funct. Anal. 98 (1991), 229-269.

[4] K.-G. Grosse-Erdmann, A. Peris Manguillot, Linear Chaos, Universitext, Springer, London, 2011.

[5] N. Nikolski, Operators, Functions, and Systems: an Easy Reading. Vol. I, Math. Surveys Monogr., Vol. 92, AMS, Providence, RI, 2002.

[6] S. Rolewicz, On orbits of elements, Studia Math. 32 (1969), 17-22.

[7] S. Shkarin, Orbits of coanalytic Toeplitz operators and weak hypercyclicity, arXiv:1210.3191.

[8] B.M. Solomyak, Cyclic families of functions for analytic Toeplitz operators, Zap. Nauchn. Sem. LOMI 157 (1987), 88-102; translation in J. Soviet Math. 44 (1989), 6, 809-819.

[9] B.M. Solomyak, A.L. Volberg, Multiplicity of analytic Toeplitz operators. In: Nikolskii N.K. (eds), Toeplitz Operators and Spectral Function Theory, Operator Theory: Advances and Applications, Vol. 42. Birkhäuser, Basel, 1989, 87-192.

Evgeny Abakumov, LAma, Univ Gustave Eiffel, Univ Paris Est Creteil, CNRS, F-77454 Marne-la-Vallée, France

Email address: evgueni.abakoumov@univ-eiffel.fr

Anton Baranov, Department of Mathematics and Mechanics, St. Petersburg State University, 28, Universitetskil Prosp., St. Petersburg, 1984504, Russia

Email address: anton.d.baranov@gmail.com

Stéphane Charpentier, Aix-Marseille Université, Centre de Mathématiques et Informatique, 39, rue F. Joliot Curie, 13453 Marseille Cedex 13, France

Email address: stephane.charpentier.10univ-amu.fr

Andrei Lishanskit, Department of Mathematics and Computer Science, St. Petersburg State University, 29B, 14 line of the Vasilievsky Island, St. Petersburg, 199178, RUSSIA

Email address: lishanskiyaa@gmail.com 